ACCEPTED MANUSCRIPT

\title{
Conductance quantization in atomic-sized gold contacts using a low cost mechanical controllable break junction setup
}

To cite this article before publication: Carla Nataly Borja Espinosa et al 2020 Eur. J. Phys. in press https://doi.org/10.1088/1361-6404/ab9fdc

\section{Manuscript version: Accepted Manuscript}

Accepted Manuscript is "the version of the article accepted for publication including all changes made as a result of the peer review process, and which may also include the addition to the article by IOP Publishing of a header, an article ID, a cover sheet and/or an 'Accepted Manuscript' watermark, but excluding any other editing, typesetting or other changes made by IOP Publishing and/or its licensors"

This Accepted Manuscript is (C) 2020 European Physical Society.

During the embargo period (the 12 month period from the publication of the Version of Record of this article), the Accepted Manuscript is fully protected by copyright and cannot be reused or reposted elsewhere.

As the Version of Record of this article is going to be / has been published on a subscription basis, this Accepted Manuscript is available for reuse under a CC BY-NC-ND 3.0 licence after the 12 month embargo period.

After the embargo period, everyone is permitted to use copy and redistribute this article for non-commercial purposes only, provided that they adhere to all the terms of the licence https://creativecommons.org/licences/by-nc-nd/3.0

Although reasonable endeavours have been taken to obtain all necessary permissions from third parties to include their copyrighted content within this article, their full citation and copyright line may not be present in this Accepted Manuscript version. Before using any content from this article, please refer to the Version of Record on IOPscience once published for full citation and copyright details, as permissions will likely be required. All third party content is fully copyright protected, unless specifically stated otherwise in the figure caption in the Version of Record.

View the article online for updates and enhancements. 


\title{
Conductance quantization in atomic-sized gold contacts using a low-cost mechanically controllable break junction setup
}

\author{
Carla Borja, ${ }^{1}$ Carlos Sabater, ${ }^{2}$ Carlos Untiedt, ${ }^{2}$ Ernesto Medina,,${ }^{1,3}$ and Werner Brämer-Escamilla ${ }^{1, *}$ \\ ${ }^{1}$ Yachay Tech University, School of Physical Sciences 8 Nanotechnology, 100119-Urcuquí, Ecuador \\ ${ }^{2}$ Departamento de Física Aplicada and Unidad asociada CSIC, \\ Universidad de Alicante, Campus de San Vicente del Raspeig, E-03690 Alicante, Spain \\ ${ }^{3}$ Simon A. Levin Mathematical, Computational and Modeling Sciences Center, \\ Arizona State University, P.O. Box 873901, Tempe, AZ 85287-3901, United States \\ (Dated: June 17, 2020)
}

\begin{abstract}
The mechanically controllable break junction (MCBJ) experimental setup is one of the main techniques employed in the study of electronic transport properties at the atomic and molecular scales. This work presents the construction of an inexpensive and simple but robust setup that shows the emergence of conductance quantization as a macroscopic gold wire is pulled to atomic dimensions. The homemade device is based on the MCBJ principle and allows forming and breaking repeatedly the metallic contact to perform a statistical analysis of the data extracting the most frequent electron transport structure. The histogram built from conductance measurements at room temperature in air, shows that the quality of the MCBJ equipment developed here is comparable to more sophisticated devices used in research laboratories. It is able to resolve up to three conductance peaks associated to gold nanowires reported in the literature. Such an experiment is suggested to be implemented as a powerful pedagogical tool in modern undergrad physics labs.
\end{abstract}

\section{INTRODUCTION}

In an attempt to understand properties of matter and quantum mechanical effects that arises as size is reduced down to the nanoscale, electrical conduction in atomicsized metallic contacts has been widely studied in a theoretical and experimental context [1]. An atomic-sized contact or nanocontact is a local point of contact between two macroscopic conductors where the electrical current is constrained to pass through a cross-section of about a nanometer. Interesting properties arise in this scenario leading to new technological implementations. In particular, it has been proposed to use single-atom contacts as quantized resistors, capacitors [2], or switches [3]. Another useful application is to fabricate nanoelectrodes to connect single molecules and characterize the electronic transport through them to be incorporated in molecular devices [4-6]. Nanowires can also be incorporated as chemical sensors due to their sensitivity to the adsorption of molecules [7].

The main experimental approaches to characterize the electronic and mechanical properties of nanocontacts are the Mechanically Controllable Break Junction (MCBJ) $[8,9]$ and the Scanning Tunneling Microscope at break junction configuration(STM-BJ) [10,11]. The principle of the MCBJ technique consists of pulling a macroscopic metal wire with a narrow section, neck or constriction. During elongation, the strain concentrates at the constriction resulting in continuous stretching of the notched section of the wire until it breaks, forming a thin contact up to the width of a single atom, before eventually breaking. Using fine control of the separation by means

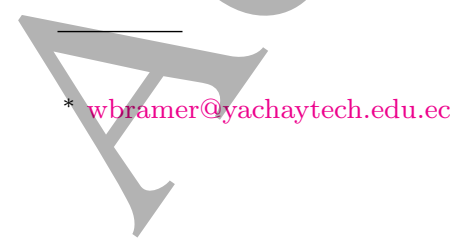

of piezoelectric materials, atomic-sized contacts can, not only, be formed and broken many times, but also stopped at an interesting configuration, in order to study e.g. I-V characteristics.

Experiments performed with MCBJ show that when the number of atoms in the center is reduced, the conductance decays [12-16]. By constructing histograms from the accumulated count of conductance values during several cycles of rupture and formation of the metallic contact [17], one observes that the junction has certain preference for integer multiples of the quantum of conductance denoted as $G_{0}=2 e^{2} / h$ where e is the electron charge and $h$ is the Planck's constant. In particular, the quantum conductance properties of gold are robust and reproducible independently of temperature, environment and experimental technique [18-20].

The phenomenon of quantization arises because of the wave characteristics of electrons. When the dimensions of the conductor are reduced and become comparable to the de Broglie wavelength of the electrons at the Fermi surface, electrons can traverse the conductor ballistically. In addition, as the width of the conductor gets narrower, the number of available electron modes is reduced and the conductance decreases in steps of the quantum of conductance [21].

We develop a simple experimental MCBJ set-up containing standard electronic parts easily found in electronics stores. Similar experimental realizations display quantized conductance steps by simply tapping on a table with two attached gold wires $[22,23]$ or using electromagnetic relays [20, 24]. However, our approach not only replaces the use of piezoelectrics that are part of conventional MCBJ, which reduces the cost of fabrication significantly but also offers finer control of the breaking and reconnecting of the wire in comparison with the abovementioned techniques. Moreover, the preparation and 
assembly of the sample do not require experience in advanced techniques such as lithography [25] and can be done by any student or professional. Finally, it includes an automatic mechanism to repeat cycles of rupture and formation continuously in order to build histograms of conductance, unlike other setups $[25,26]$ that only allow observing individual traces of conductance.

The building of the device is useful for amateur enthusiasts, advanced high school students or for lab courses at the undergrad level. Directly appreciating a quantum property using an experimental setup at room temperature will be a fascinating demonstration of the relevance of quantum mechanics in our daily lives. In the context of basic Nanotechnology, it will also illustrate the natural deviation of conductance from classical Ohm's law at the nanoscale. Finally, it is also a good way to get students interested in electronic setups for data acquisition. Even a person with a very basic physics background will understand the underpinnings of the physics that lies at the nanoscale.

\section{BREAK JUNCTION}

\section{A. Experimental setup}

The break junction setup consists of a lever with a long $(109 \mathrm{~cm})$ and a short arm $(6.5 \mathrm{~cm})$ joined at a pivot, (see Figure 1(a)). The lengths are chosen so that a fraction of $\mathrm{cm}$ displacements made periodically by a loudspeaker on one end, turn into micro-metric stable displacements on the other end e.g., a displacement of $0.1 \mathrm{~cm}$ of the long arm would correspond to a displacement of $60 \mu \mathrm{m}$ on the opposite arm. On the short arm, the end of the lever bends a steel strip containing the nanocontact.

As shown in Figure 1(c), the sample holder design consists of a three-point bending structure (shown at the right bottom) formed by two mobile metal rods placed $10 \mathrm{~cm}$ apart from each other and a metal pushrod in between. The vertical position of the lateral supports is controlled by turning the central screw that controls the z-position of the structure. The pushrod is fixed to an aluminum rail whose opposite end rests over a loudspeaker of 8 Ohms impedance and 8 inches of diameter (shown on the left bottom of Figure 1 (b)). The rail can pivot about a point $6.5 \mathrm{~cm}$ away from the pressure point of the pushrod over the sample substrate. The vibrations from the loudspeaker move the end of the rail upward and downward. Therefore, these vibrations are the ones that control the displacement of the pushrod vertically and as a consequence, the bending or relaxation of the steel substrate, or in other words, control the formation and breaking mechanism of the metallic contact.

Figure 2 shows the steps needed in order to prepare the sample. First, a $10 \times 1 \mathrm{~cm}$ steel spring substrate of $4 \mathrm{~mm}$ in thickness is covered with insulating Kapton tape (Figure 2 (a)-(b)). Second, a gold wire (high purity 4n) $2 \mathrm{~cm}$ long with a neck or constriction at the center,

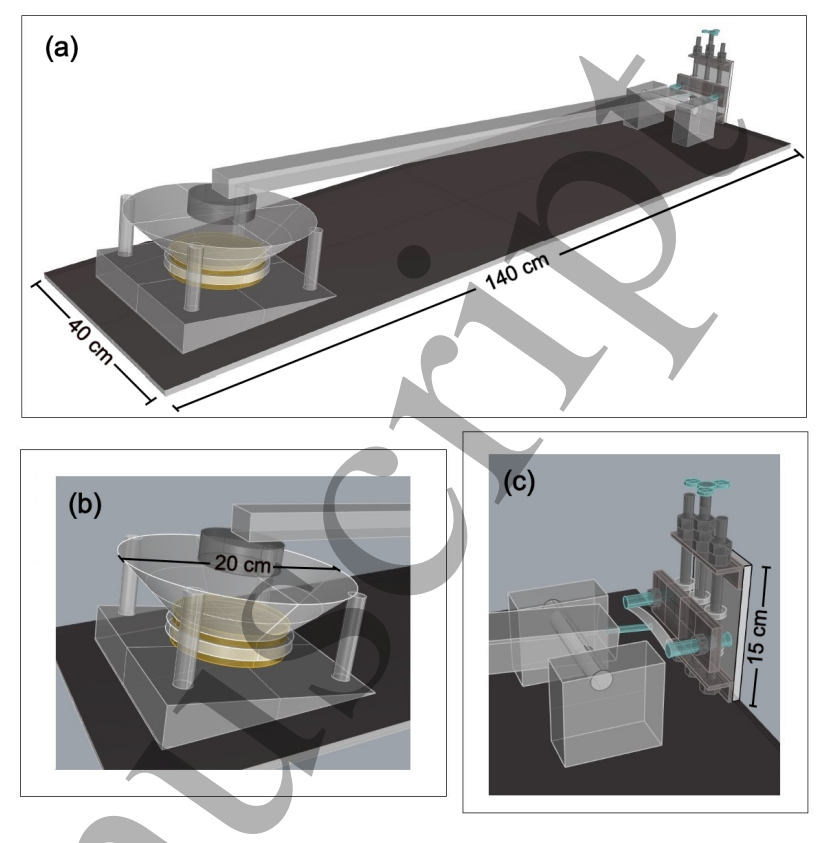

FIG. 1. Mechanically Controlled Break Junction setup, consisting of a lever with a long and a short arm so that a fraction of $\mathrm{cm}$ displacements (by loudspeaker) turn into micro-metric stable displacements on the other arm (steel bending strip). a) The main system structure showing the loudspeaker and the coarse control mechanism on both sides, b) loudspeaker that acts as the periodic motion device, c) the stainless steel bending sheet sample holder structure with the coarse control mechanism. The plastic dish at the end the screw is used for a better control of the lifting system.

is fixed to the steel substrate using nail polish (Figure $2(\mathrm{c}))$. The constriction in the wire is previously cut, at approximately one-third of the wire width, by rolling the wire under a surgical knife. Third, two triangularshaped drops of epoxy adhesive are applied very close to the constriction without touching each other leaving in between the neck of the wire (Figure 2 (d)). Figure 3 shows a close up view of wire neck between the facing epoxy drops. Finally, both ends of the gold wire are welded to copper wires using silver welding (Figure 2 (e)).

\section{B. Electronics}

Figure 4 shows the overall circuit used for the operation of the equipment. A voltage divider with a variable resistor adjusts the bias voltage for the break-junction. Then, the operational amplifier (op-amp) LM358P connected to a feedback resistor of $R_{f}=100 K \Omega$ and a feedback capacitor of $22 \mathrm{pF}$ amplifies the signal and converts the current to voltage. The output signal from the op-amp goes to the analog input of a National Instrument data acquisition DAQ device (NI USB-6210 16-bit), using a coaxial cable to reduce noise and interference from the environment. The wave generator based on the model XR2206 integrated circuit, sends a sinusoidal signal to 


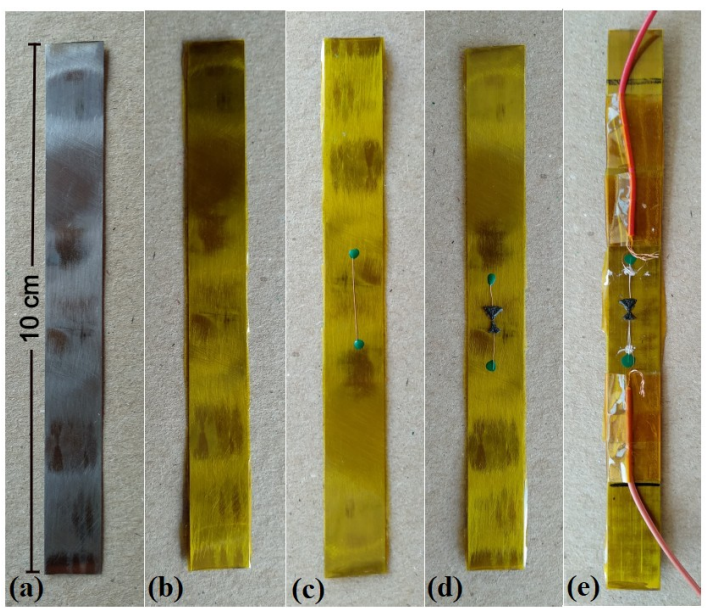

FIG. 2. Top view of the sample preparation process. (a) Initial steel sheet substrate. (b) Steel sheet covered with insulating Kapton tape. (b) Neck-shaped gold wire fixed at the ends to the steel substrate with nail polish. (d) Two drops of epoxy adhesive applied one at each side of the constriction in the wire. (e) Copper wires welded to both ends of the gold wire using silver welding.

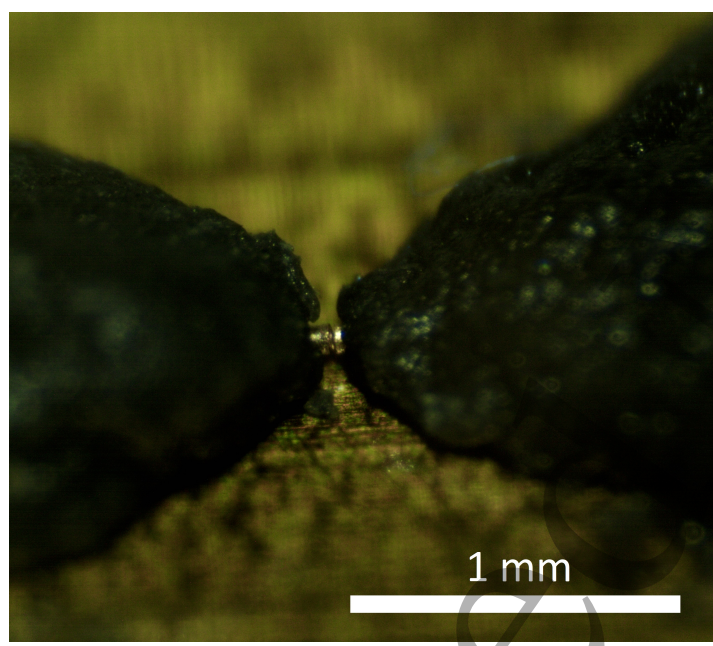

FIG. 3. Detail of contact once two ends of wire are fixed to the bending strip with epoxy. The wire shows the conic dent made by the guillotine.

the audio amplifier TDA7293 that is connected to a $110 \mathrm{~V}$ $\mathrm{AC}$ transformer to control the frequency and amplitude of the vibrations of the loudspeaker.

To activate the trigger or acquisition of the data, a voltage comparison module compares the voltage from the sinusoidal wave sent to the audio amplifier, after passing through a voltage divisor, with a reference value set by the voltage at the inverting input of the op-amp LM741CP. When the voltage coming from the sinusoidal wave is higher than the reference value, the output from the op-amp will be + Vcc; the DAQ board reads this value and a LabVIEW program on the computer orders the DAQ to start acquiring the data. By modifying the ref-

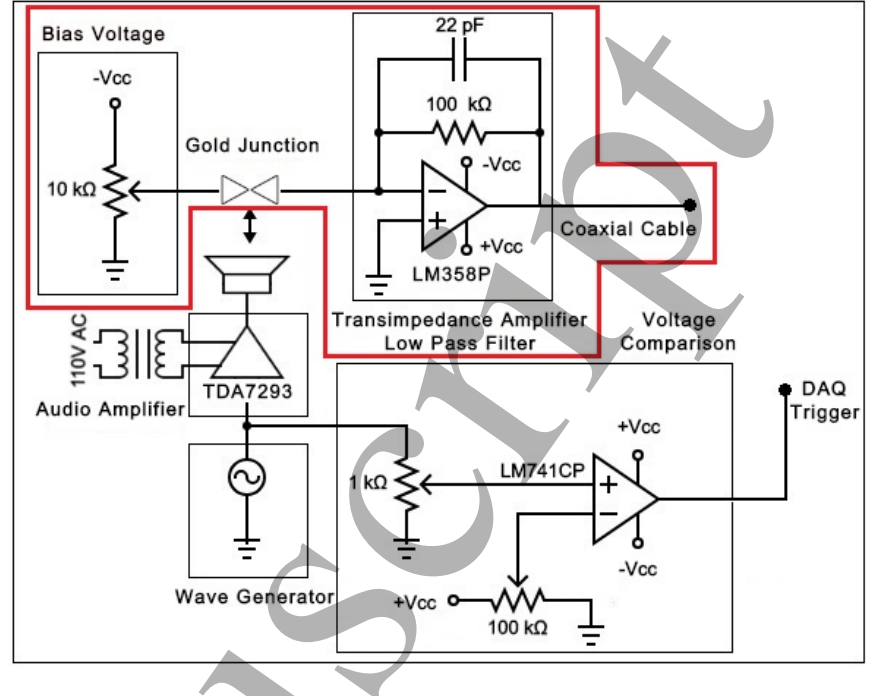

FIG. 4. Circuit diagram that shows the electronic components and connections of the MCBJ equipment. The op-amps are powered using a symmetric power supply of $V_{c c}= \pm 6 \mathrm{~V}$. All the potentiometers are multi-turn for higher sensitivity. The wave generator is a low cost XR2206 1HZ-1MHZ Kit.

erence voltage, the activation of the trigger is set close to the moment of rupture and formation of the atomic-sized contact. All the electric components are placed on the same/breadboard and a symmetric power supply, powers the circuit. A LabVIEW program on the computer shows the traces of voltage as a function of time and the progressive construction of the histogram during the experiment. It also saves the data to a text file for further analysis.

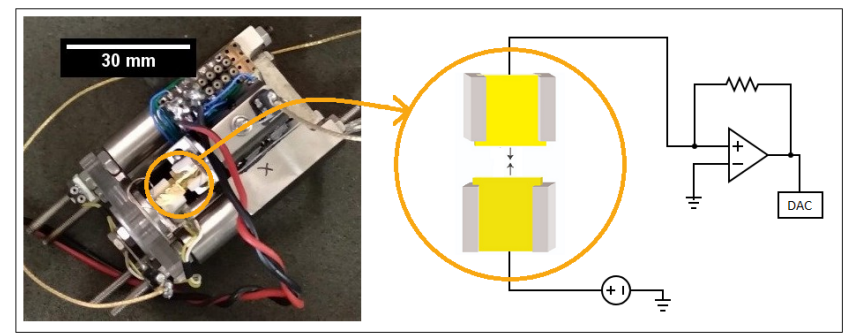

FIG. 5. STM microscope in the break junction regime used to measure conductance through atomic-sized contacts built in the LT-Nanolab of the University of Alicante (left). Representation of the circuit and type of electrodes used to form the contact (right).

\section{MEASUREMENT}

In order to see the quality of operation of our equipment, the results were compared with those obtained from a research grade device built at the "Laboratorio de Bajas Temperaturas y Sistemas Nanométricos" at the 

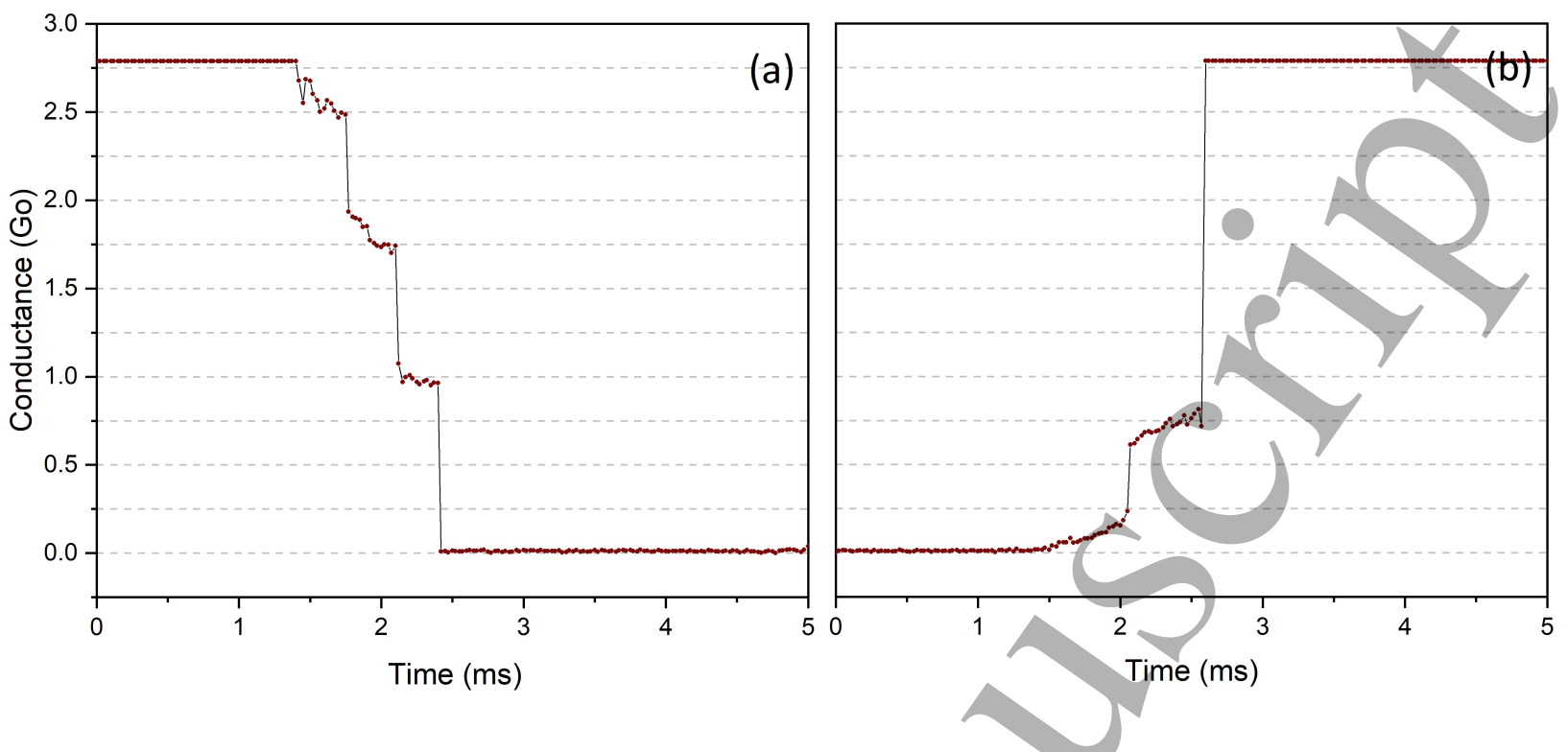

FIG. 6. Two conductance traces obtained with the MCBJ device

at room temperature with a bias voltage of $220 \mathrm{mV}$. (a) Conductance of a single junction rupture as the contact is pulled. Three quantized conductance steps are clearly identified. (b) Conductance of a single junction formation trace by indentation. The exponential conductance increase from zero to $2 \mathrm{~ms}$ corresponds to tunneling processes.

University of Alicante Spain, using a STM-BJ. It employs two gold sheets as electrodes to form the contact. The sheets of $0.5 \times 0.5 \mathrm{~cm}$ long and $100 \mu \mathrm{m}$ of thickness fit on support structures, as shown in Figure 5 to the right. These supports are twisted with respect to each other and fixed, one to a mobile part in the head of the microscope and the other one to the base. The mobile part of the microscope, at which one of the electrodes is fixed, rests on four piezo piles, that allow it to move perpendicular to the base of the microscope.

\section{A. Conductance traces}

Conductance traces consist in measuring the conductance as function of time for an atomic bridge while changing its length and thickness. This bridge can be formed by elongation or indentation of the two metallic contacts. The maximum conductance $n_{0}$ in units of Go that our equipment can measure is obtained from circuit analysis (the part inside the red lines in Figure 4). The following relation applies

$$
n_{0}=\frac{1}{\frac{V_{\text {Bias }}}{V_{\text {out }}} \frac{R_{f}}{R_{G_{0}}}+\frac{R_{i}}{R_{G_{0}}}},
$$

where $V_{\text {out }}$ is the maximum output voltage from the operational amplifier (op-amp), $V_{\text {Bias }}$ is the voltage applied to the break-junction, $R_{f}$ is the feedback resistor $(100 k \Omega)$, $R_{G_{0}}$ is the resistance that corresponds to one quantum of conductance $(\approx 12.9 k \Omega)$, and $R_{i}$ is the internal resistance of the bias voltage supply. For the case when $R_{i} \ll R_{G_{0}}$

$$
n_{0}=\frac{V_{\text {out }}}{V_{\text {Bias }}} \frac{R_{G_{0}}}{R_{f}} .
$$

For our setup $V_{\text {out }}=4.75 \mathrm{~V}, V_{\text {Bias }}=220 \mathrm{mV}, R_{f}=$ $100 k \Omega, R_{i} \simeq 0 \Omega$, therefore, the setup can measure up to $2.78 G_{0}$. The maximum output voltage of $4.75 \mathrm{~V}$ is limited by the voltage supply $V_{c c}$ of $6 \mathrm{~V}$ applied to the operational amplifier. Increasing the voltage supply would increase the range of conductance $n_{0}$. However, a higher $V_{c c}$ value introduces electric noise to the components on the breadboard, producing irregularities at the base voltage signal and conductance values significantly out of the flat range within the plateaus in the traces of conductance. Taking the above into consideration, we set the voltage supply no higher than $6 \mathrm{~V}$, and the bias voltage at $220 \mathrm{mV}$ in such a way that the output voltage corresponding to $1 G_{0}$ equals $1.7 \mathrm{~V}$, relatively far from the base noise signal and we still can observe the third conductance peak in the histogram of gold. At ambient conditions the three main peaks of the conductance histogram of gold are placed at: $0.921 G_{0}, 1.771 G_{0}, 2.682 G_{0}$ [27].

Figure 6 shows individual conductance traces measured with the MCBJ equipment. Each trace curve has 200 measurements of conductance took in a lapse of 5 ms $(25 \mu$ s between consecutive points of measurement). The error in the conductance is around $\pm 0.02 G_{0}$. Curve (a) shows how the conductance decreases in three steps as the electrodes move apart with plateaus close to 1 , 2 and $3 G_{0}$. Curve (b) illustrates the process of contact formation. As the electrodes get closer the conduc- 

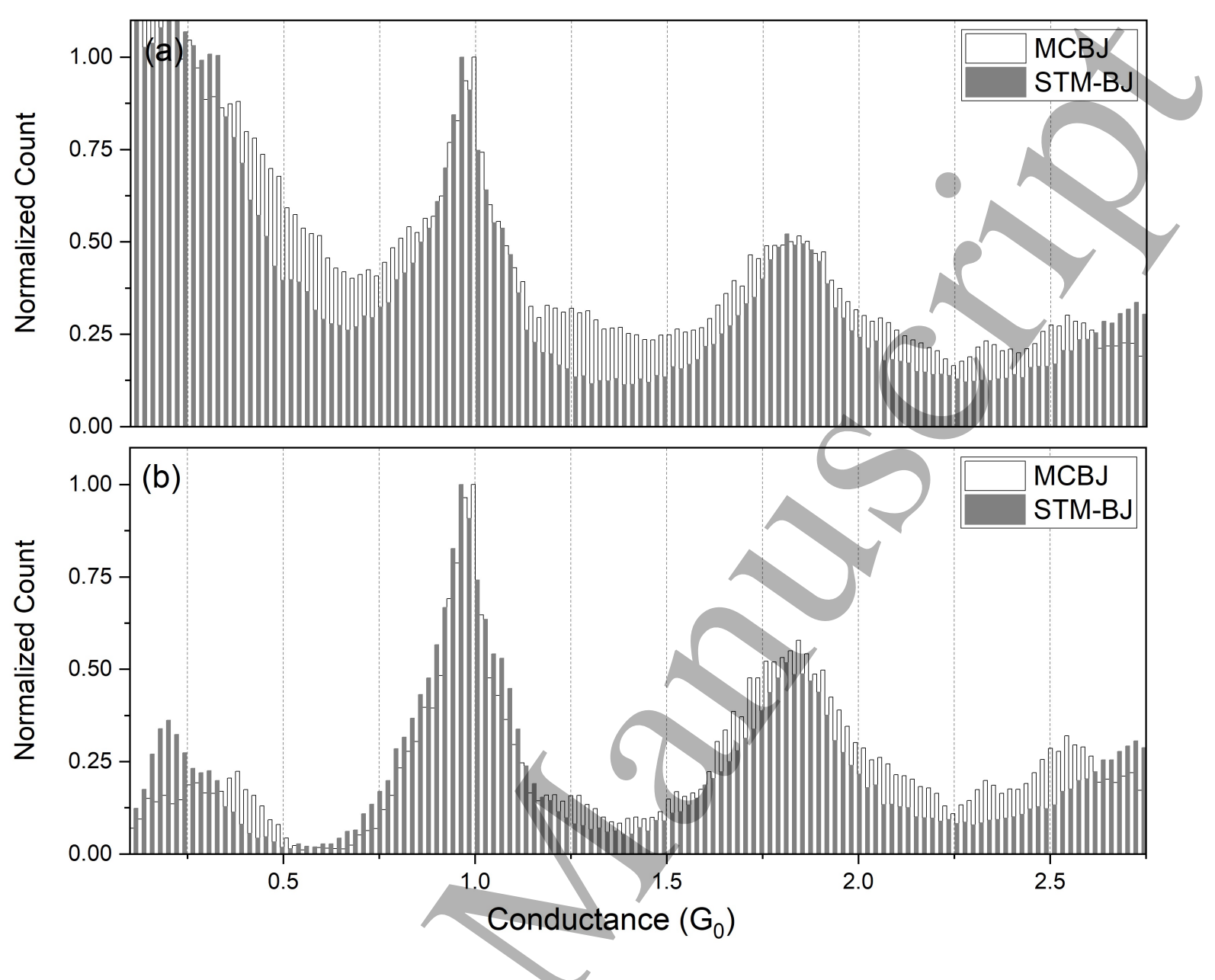

FIG. 7. Comparison of the measurements performed with the MCBJ (no-filled histogram) and STM-BJ (gray-filled histogram) setups. (a) Histograms built from accumulated counts of rupture and formation events. (b) Histograms built from accumulated events only from rupture process.

tance increases exponentially (this corresponds to tunneling conduction) and suddenly jumps to contact below $1 G_{0}$, after which it reaches the maximum conductance. In order to create conductance histograms, it is necessary to measure a high number of conductance traces like the ones shown in Figure 6. We neglected the traces that do not represent a complete cycle of rupture (to reach the base signal from the maximum output voltage) or complete cycle of formation (to reach the maximum output voltage from the base signal).

\section{B. Histograms}

Figure 7 shows the histograms built from accumulated traces obtained with the STM-BJ (gray-filled histogram) and MCBJ (no-filled histogram) techniques. The panel (a) corresponds to the accumulated counts taking into account rupture and formation events. The panel (b) corresponds to the accumulated events only from rupture processes. The MCBJ histograms (no-filled) were built with conductance curves captured after 5349 and 1188 trigger activation events for (a) and (b) respectively.

Regardless of the technique (STM-BJ or MCBJ), the conductance histograms of Figure 7 show three main peaks. For the MCBJ histogram the peak mean value positions are $0.981 \pm 0.005 G_{0}, 1.83 \pm 0.01 G_{0}, 2.57 \pm$ $0.02 G_{0}$ (based on a Gaussian fit not shown in the Figure), and correspond to the conductance quantization in gold nanowires. The tunneling contribution from contact formation events can be appreciated in the increased counts below the peak at $1 G_{0}$ in Figure 7 (a). On the other hand, panel (b) shows a broad peak at non-integer values below $0.5 G_{0}$, positioned at $0.31 \pm 0.03 G_{0}$ for the MCBJ (no-filled) histogram. This peak suggests that the sample is not clean due to the presence of adsorbents from atmospheric contamination. Moreover, the peaks appear broader than the typical conductance histogram of gold reported in the literature which is indicative of thermal effects. 
We want to point out, as shown in Figure 7, that the main features of the histograms accumulated from measurements taken with our MCBJ setup are similar to those taken by a research grade device driven by a high quality piezoelectric (STM-BJ), indicating the proper operation and accuracy of the built equipment. Note that the structure of the peaks is the same and the positions of the peaks fall within the quantum conductance range corresponding to gold nanowires. The main difference between the MCBJ and STM-BJ histograms is the peak below 0.5 Go attributed to adsorbates and contaminants that depend on the ambient conditions of each laboratory. The reproducibility of the exact shape of the conductance histogram, even by using the same sample and instrument, is affected among other factors by the crystallinity of the sample, mechanical and electrical noise. The last factor has a major effect on our setup since our circuit has no filters. This is manifested in the increased counts at the valleys of the histogram.

\section{Summary and conclusions}

This work described the construction of an innovative, robust, low-cost and simple device to measure conductance through atomic-sized contacts of gold, that is able to obtain statistical information of the experimental data by using a DAQ. The resolution of the built equipment is comparable to that of a research grade device, such as the STM-BJ built in the LT-Nanolab of the University of Alicante. Nevertheless, the access to higher conductance peaks is limited in our equipment due the level of electronic noise.

Our device has the advantage that it replaces the use of piezoelectric materials that are part of conventional types of MCBJ, with the action of the vibrations of a loudspeaker, which reduces the cost of fabrication and miniaturization significantly, therefore being more accessible to incorporate into undergraduate advanced physics programs.

\section{ACKNOWLEDGMENT}

We are gratefully for all the support of Generalitat Valenciana through PROMETEO2017/139 and GENT
(CDEIGENT2018/028). We are gratefully to Prof. Oren Tal from Weizmann Institute of Science for his valuable collaboration in providing his laboratory facilities for training in the MCBJ technique. This work was supported by CEPRA XII Grant XII-2018-06 "Mechanical Spectroscopy".

APPENDIX A: LABVIEW PROGRAM TO OBTAIN VOLTAGE TRACES FROM THE MCBJ

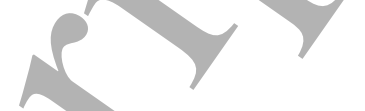

For the acquisition of the data, we use a National Instruments data acquisition device (DAQ), NI USB-6210. The operation of the DAQ is controlled with a LabVIEW program shown in Figure 8, where red enumerated boxes mark the main parts of the program. These parts are shown separately in Figure 9.

Red box (1) contains four DAQmx modules. The 'AI voltage' DAQmx defines the voltage range to be measured. The 'Sample Clock' DAQmx sets the sampling rate and the number of samples to acquire. The following DAQmx establishes the activation of the trigger depending on the rising or falling slope of the digital signal. The last DAQmx uploads the previous parameters programming to the DAQ device. Red box (2) consists of a DAQmx module responsible for reading the data. Red box (3) takes the voltage values from Red box (2) and assigns a time value to each data point according to the delta time $\left(2.5 \times 10^{-5} s\right)$. Red box (4) creates an array with equally spaced time values corresponding to the moment of acquisition of each data point since it is equal for all traces, the array is created one time during the first iteration of the while loop. Red box (5) creates an array with the incoming voltage measurements for each trace, limiting the values to the range between Vmin and $V \max$, which are set according to the user's preference. Red box (6) allows the user to save the data to a .txt file when deemed appropriate. Red box (7) creates a two dimensional array from the accumulated measurements, where each column represents a trace curve. It also allows the user to clean the register in order to accumulate a new group of traces. Red box (8) creates the corresponding histogram and displays it in the front panel. Finally, red box (9) selects an individual trace curve and displays it in the front panel.
[1] Nicolás Agraït, Alfredo Levy Yeyati, and Jan M Van Ruitenbeek. Quantum properties of atomic-sized conductors. Physies Reports, 377(2-3):81-279, 2003.

[2] Jian Wang, Hong Guo, Jose-Luis Mozos, CC Wan, Gianni Taraschi, and Qingrong Zheng. Capacitance of atomic junctions. Physical Review Letters, 80(19):4277, 1998.

[3] K Terabe, T Hasegawa, T Nakayama, and M Aono. Quantized conductance atomic switch. Nature,
433(7021):47-50, 2005

[4] Xiao-Shun Zhou, Jing-Hong Liang, Zhao-Bin Chen, and Bing-Wei Mao. An electrochemical jump-to-contact stmbreak junction approach to construct single molecular junctions with different metallic electrodes. Electrochemistry Communications, 13(5):407-410, 2011.

[5] Bingqian Xu and Nongjian J Tao. Measurement of singlemolecule resistance by repeated formation of molecular junctions. Science, 301(5637):1221-1223, 2003. 
[6] Nongjian Tao. Electron transport in molecular junctions. Nature, 1:173, 2006.

[7] CZ Li, HX He, A Bogozi, JS Bunch, and NJ Tao. Molecular detection based on conductance quantization of nanowires. Applied Physics Letters, 76(10):1333-1335, 2000.

[8] C.J. Muller, J.M. van Ruitenbeek, and L.J. de Jongh. Experimental observation of the transition from weak link to tunnel junction. Physica C, 191(3):485 - 504, 1992.

[9] C. J. Muller, J. M. van Ruitenbeek, and L. J. de Jongh. Conductance and supercurrent discontinuities in atomicscale metallic constrictions of variable width. Phys. Rev. Lett., 69:140-143, Jul 1992.

[10] J. I. Pascual, J. Méndez, J. Gómez-Herrero, A. M. Baró, N. García, and Vu Thien Binh. Quantum contact in gold nanostructures by scanning tunneling microscopy. Phys. Rev. Lett., 71:1852-1855, Sep 1993.

[11] N. Agraït, J. G. Rodrigo, and S. Vieira. Conductance steps and quantization in atomic-size contacts. Phys. Rev. B, 47:12345-12348, May 1993.

[12] N Agraï, JG Rodrigo, and S Vieira. Conductance steps and quantization in atomic-size contacts. Physical Review $B, 47(18): 12345,1993$.

[13] L Olesen, E Laegsgaard, I Stensgaard, F Besenbacher, J Schio/tz, P Stoltze, KW Jacobsen, and JK No/rskov. Quantized conductance in an atom-sized point contact. Physical Review Letters, 72(14):2251-2254, April 1994.

[14] JM Krans, JM Van Ruitenbeek, VV Fisun, IK Yanson, L De Jongh, and J. The signature of conductance quantization in metallic point contacts. Nature, 375(6534):767, 1995.

[15] JI Pascual, J Mendez, J Gomez-Herrero, AM Baro, N Garcia, Uzi Landman, WD Luedtke, EN Bogachek, and H-P Cheng. Properties of metallic nanowires: from conductance quantization to localization. Science, 267(5205):1793-1795, 1995.

[16] Zheng Gai, Yi He, Hongbin Yu, and/WS Yang. Observation of conductance quantization of ballistic metallic point contacts at room temperature. Physical Review B,
53(3):1042, 1996.

[17] J. M. Krans, J. M. van Ruitenbeek, N. V. Fisun, I. K. Yanson, and L. J. de Jongh. The signature of conductance quantization in metallic point contacts. 375(6534):767-769, 1995.

[18] JL Costa-Krämer, N García, P García-Mochales, PA Serena, MI Marqués, and A Correia. Conductance quantization in nanowires formed between micro and macroscopic metallic electrodes. Physical Review B, 55(8):5416, 1997.

[19] JL Costa-Krämer. Conductance quantízation at room temperature in magnetic and nonmagnetic metallic nanowires. Physical Review B, 55(8):R4875, 1997.

[20] K Hansen, Erik Lægsgaard, Ivan Stensgaard, and Flemming Besenbacher. Quantized conductance in relays. Physical Review B, 56(4):2208, 1997.

[21] Yuli Nazarov. Quantum transport : introduction to nanoscience. Cambridge University Press, 2009.

[22] JL Costa-Krämer, N Garcia, P Garcia-Mochales, and PA Serena. Nanowire formation in macroscopic metallic contacts: quantum mechanical conductance tapping a table top. Surface Science, 342(1-3):L1144-L1149, 1995.

[23] EL Foley, D Candela, KM Martini, and MT Tuominen. An undergraduate laboratory experiment on quantized conductance in nanocontacts. American Journal of Physics, 67(5):389-393, 1999.

[24] Frederic Ott and James Lunney. Quantum conduction: a step-by-step guide. Europhysics news, 29(1):13-16, 1998.

[25] EH Huisman, FL Bakker, JP van der Pal, RM de Jonge, and $\mathrm{CH}$ van der Wal. Public exhibit for demonstrating the quantum of electrical conductance. American Journal of Physics, 79(8):856-860, 2011.

[26] R Tolley, A Silvidi, C Little, and KF Eid. Conductance quantization: A laboratory experiment in a senior-level nanoscale science and technology course. American Journal of Physics, 81(1):14-19, 2013.

[27] These values were obtained at the LT-Nanolab of the University of Alicante. 

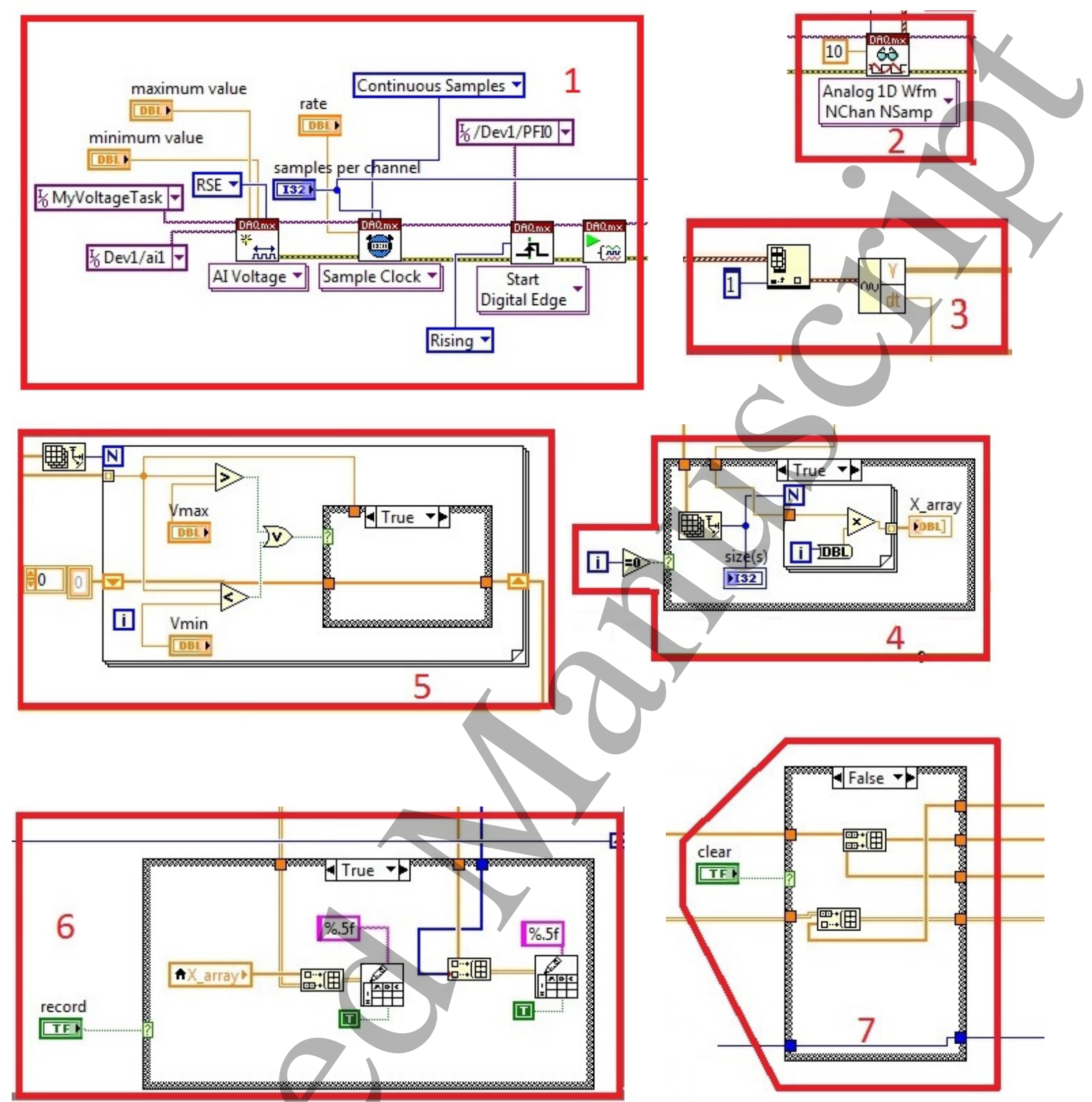

FIG. 9. Details of the main parts of the LabView program. (1) Main set up parameters. (2) Data acquisition. (3) Relation of voltage values with time steps (4) Creation of the time column for all traces. (5) Creation of an array with voltage data from each trace within a specific range. (6) Recording of all the data to a .txt file. (7) Accumulation of traces in a two dimensional array. (8) Creation of the histogram to be shown in the front panel. (9) Selection of an individual trace curve to be shown in the front panel. 